Теорія Ймовір. та Матем. Статист. Вип. 68, 2003
Theor. Probability and Math. Statist. S 0094-9000(04)00590-3

Article electronically published on May 11, 2004

\title{
AN ESTIMATE OF THE RATE OF CONVERGENCE TO THE NORMAL LAW
}

UDC 519.21

\author{
I. YO. POLYAK
}

Abstract. The paper contains an estimate of the rate of convergence to the normal law for nonidentically distributed random variables.

A generalization of a V. M. Zolotarev result for the case of nonidentically distributed random variables is obtained in 1]. This paper continues the line of research initiated in [1] on applications of pseudomoments to the rate of convergence to the normal law. However below we use other pseudomoments different from those used in 11.

Let $\xi_{1}, \xi_{2}, \ldots, \xi_{n}, \ldots$ be a sequence of independent random variables such that $\mathrm{M} \xi_{i}=0$ and $\operatorname{Var} \xi_{i}=\sigma_{i}^{2}$. Let $F_{i}(x)$ and $f_{i}(t)$ be the distribution function and characteristic function of the random variable $\xi_{i}, i \geq 1$, respectively. Denote by $\Phi_{n}(x)$ the distribution function of the random variable $\left(\xi_{1}+\xi_{2}+\cdots+\xi_{n}\right) / B_{n}$ and put $B_{n}^{2}=\sigma_{1}^{2}+\cdots+\sigma_{n}^{2}$, $\bar{\sigma}_{i}=\min \left(1 ; \sigma_{i}\right)$, and $\bar{B}_{n}^{2}=\bar{\sigma}_{1}^{2}+\cdots+\bar{\sigma}_{n}^{2}$. Further let $\Phi(x)$ be the standard normal distribution function, $\rho_{n}=\sup _{x}\left|\Phi_{n}(x)-\Phi(x)\right|$, and

$$
\begin{gathered}
\lambda_{n i}^{(1)}=\int_{|x| \leq B_{n}} \max \left(1,|x|^{3}\right)\left|d\left(F_{i}(x)-\Phi\left(\frac{x}{\sigma_{i}}\right)\right)\right|, \\
\lambda_{n i}^{(2)}=\int_{|x| \geq B_{n}} x^{2}\left|d\left(F_{i}(x)-\Phi\left(\frac{x}{\sigma_{i}}\right)\right)\right|, \\
\Lambda_{n}^{(1)}=\frac{1}{\bar{B}_{n}^{2}} \sum_{i=1}^{n} \lambda_{n i}^{(1)}, \quad \Lambda_{n}^{(2)}=\frac{1}{\bar{B}_{n}^{2}} \sum_{i=1}^{n} \lambda_{n i}^{(2)}, \quad b_{n}=\min \left(\bar{\sigma}_{1}, \ldots, \bar{\sigma}_{n}\right) .
\end{gathered}
$$

Theorem. For all $n \geq 1$,

$$
\rho_{n} \leq C\left(\frac{1}{\bar{B}_{n}} \Lambda_{n}^{(1)}+\Lambda_{n}^{(2)}\right) b_{n}^{-3}
$$

where $C$ is a universal constant.

Proof. Let $n$ be fixed and $c \in(0 ; 1)$ be a certain constant; the exact value of $c$ will be chosen below. Assume that $\Lambda_{n}^{(2)} \leq c$ and note that estimate (1) is obvious otherwise.

For $n=1$ we have

$$
\begin{aligned}
\rho_{1} & =\sup _{x}\left|F_{1}(x \sigma)-\Phi(x)\right|=\sup _{x}\left|F_{1}(x)-\Phi\left(x / \sigma_{1}\right)\right|=\sup _{x}\left|\int_{-\infty}^{+\infty} d\left(F_{1}(x)-\Phi\left(x / \sigma_{1}\right)\right)\right| \\
& \leq \int_{-\infty}^{+\infty}\left|d\left(F_{1}(x)-\Phi\left(x / \sigma_{1}\right)\right)\right| \leq \lambda_{n 1}^{(1)}+\frac{1}{\sigma_{1}^{2}} \lambda_{n 1}^{(2)},
\end{aligned}
$$

and inequality (1) follows. Now let $n \geq 2$.

2000 Mathematics Subject Classification. Primary 60F10. 
Put $\phi_{n}(t)=\prod_{i=1}^{n} f_{i}\left(t / B_{n}\right)$. We apply the following inequality:

$$
\rho_{n} \leq \min \left\{\max \left(\int_{0}^{X}\left|\phi_{n}(t)-e^{-t^{2} / 2}\right| \frac{d t}{t}, \frac{6 \sqrt{2}}{X}\right) ; X>0\right\}
$$

(see [2] p. 377]) with $X=T B_{n}, T=c^{5 / 2} \Lambda_{n}^{-1}$, and $\Lambda_{n}=\max \left(\Lambda_{n}^{(1)} ; \Lambda_{n}^{(2)}\right)$. Let $T_{1}=\sqrt{c}$, $T_{2}=\min \left(T_{1}, T\right)$. Using the inequalities

$$
\left|\phi_{n}(t)-e^{-t^{2} / 2}\right| \leq \sum_{i=1}^{n}\left|\omega_{i}\left(\frac{t}{B_{n}}\right)\right|\left|\psi_{n i}(t)\right|,
$$

where $\omega_{i}(t)=f_{i}(t)-\exp \left\{-t^{2} \sigma_{i}^{2} / 2\right\}, \psi_{n i}(t)=\prod_{k=1}^{i-1} \exp \left\{-t^{2} \sigma_{k}^{2} / 2 B_{n}^{2}\right\} \prod_{k=i+1}^{n} f_{k}\left(t / B_{n}\right)$, and

$$
\left|\omega_{i}(t)\right| \leq \min \left(\frac{|t|^{3}}{6} \lambda_{n i}^{(1)}+2 t^{2} \lambda_{n i}^{(2)} ; \lambda_{n i}^{(1)}+\frac{1}{B_{n}^{2}} \lambda_{n i}^{(2)}\right),
$$

we get

$$
\begin{aligned}
\left|f_{k}(t)\right| & \leq\left|\exp \left\{-t^{2} \sigma_{k}^{2} / 2\right\}+\omega_{k}(t)\right| \leq \exp \left\{-t^{2} \sigma_{k}^{2} / 2\right\}+\frac{\left|t^{3}\right|}{6} \lambda_{n k}^{(1)}+2 t^{2} \lambda_{n k}^{(2)} \\
& \leq \exp \left\{-t^{2} \bar{\sigma}_{k}^{2} / 2\right\}+t^{2}\left(\frac{c^{5 / 2}}{6}+2 c\right) \leq \exp \left\{-c_{1} t^{2} \bar{\sigma}_{k}^{2}\right\}
\end{aligned}
$$

for $|t| \leq T_{2}$, while

$$
\left|\psi_{n i}(t)\right| \leq e \cdot \exp \left\{-t^{2} \frac{\bar{B}_{n}^{2}}{B_{n}^{2}} c_{1}\right\}
$$

for $|t| \leq T_{2} B_{n}$, where $c_{1}=\left(1-e^{-c / 2}\right) / c-13 c / 6$ and $c$ is chosen such that $c_{1}>0$. Then (see inequality (12) in 1])

$$
\left|\phi_{n}(t)-e^{-t^{2} / 2}\right| \leq e\left(\frac{|t|^{3}}{6} \Lambda_{n}^{(1)}+2 t^{2} \Lambda_{n}^{(2)}\right) \exp \left\{-t^{2} \frac{\bar{B}_{n}^{2}}{B_{n}^{2}} c_{1}\right\} .
$$

We represent the integral in (2) as follows:

$$
\begin{aligned}
I & =\int_{0}^{T B_{n}}\left|\phi_{n}(t)-e^{-t^{2} / 2}\right| \frac{d t}{t} \\
& =\int_{0}^{T_{2} B_{n}}\left|\phi_{n}(t)-e^{-t^{2} / 2}\right| \frac{d t}{t}+\int_{T_{2} B_{n}}^{T B_{n}}\left|\phi_{n}(t)-e^{-t^{2} / 2}\right| \frac{d t}{t}=I_{1}+I_{2} .
\end{aligned}
$$

Inequality (3) implies

$$
I_{1}=\int_{0}^{T_{2} B_{n}}\left|\phi_{n}(t)-e^{-t^{2} / 2}\right| \frac{d t}{t} \leq C^{(1)}\left(\frac{1}{\bar{B}_{n}} \Lambda_{n}^{(1)}+\Lambda_{n}^{(2)}\right) .
$$

The integral $I_{2}$ is nonzero if $T_{2}=T_{1}$, in which case $\Lambda_{n}^{(1)} \leq c^{2}$. Thus

$$
\begin{aligned}
&\left|f_{i}\left(\frac{t}{B_{n}}\right)\right| \leq \exp \left\{-t^{2} \sigma_{i}^{2} /\left(2 B_{n}^{2}\right)\right\}+\left|\omega_{i}\left(\frac{t}{B_{n}}\right)\right| \\
& \leq \exp \left\{\left(e^{-c / 2}-1\right) \bar{\sigma}_{i}^{2}+\lambda_{n i}^{(1)}+\frac{1}{B_{n}^{2}} \lambda_{n i}^{(2)}\right\}, \\
& \prod_{\substack{k=1 \\
k \neq i, j}}^{n}\left(\exp \left\{-t^{2} \sigma_{1}^{2} /\left(2 B_{n}^{2}\right)\right\}+\left|\omega_{k}\left(\frac{t}{B_{n}}\right)\right|\right) \leq e^{2+c} \exp \left\{-\left(\frac{1-e^{-c / 2}}{c}-c\right) c \bar{B}_{n}^{2}\right\}
\end{aligned}
$$


for $T_{2} B_{n} \leq|t| \leq T B_{n}$. Moreover by inequality (13) in [1],

$$
\begin{aligned}
\left|\phi_{n}(t)-e^{-t^{2} / 2}\right| \leq & \sum_{i=1}^{n}\left|\omega_{i}\left(\frac{t}{B_{n}}\right)\right| \sum_{\substack{j=1 \\
j \neq i}}^{n} e^{-t^{2} \sigma_{j}^{2} /\left(2 B_{n}^{2}\right)} \prod_{\substack{k=1 \\
k \neq i, j}}^{n}\left(e^{-t^{2} \sigma_{k}^{2} /\left(2 B_{n}^{2}\right)}+\left|\omega_{k}\left(\frac{t}{B_{n}}\right)\right|\right) \\
& +\prod_{k=1}^{n}\left|\omega_{k}\left(\frac{t}{B_{n}}\right)\right| \\
\leq & \sum_{i=1}^{n}\left|\omega_{i}\left(\frac{t}{B_{n}}\right)\right| e^{-t^{2} b_{n}^{2} /\left(2 B_{n}^{2}\right)}(n-1) e^{2+c} \exp \left\{-c_{3} c \bar{B}_{n}^{2}\right\} \\
& +\prod_{k=1}^{n}\left(\lambda_{n k}^{(1)}+\frac{1}{B_{n}^{2}} \lambda_{n k}^{(2)}\right),
\end{aligned}
$$

where $c_{3}=\left(1-e^{-c / 2}\right) / c-c>0$.

Thus

$$
\begin{aligned}
I_{2}= & \int_{T_{2} B_{n}}^{T B_{n}}\left|\phi_{n}(t)-e^{-t^{2} / 2}\right| \frac{d t}{t} \\
\leq & (n-1) e^{2+c} \exp \left\{-c_{3} c \bar{B}_{n}^{2}\right\} \sum_{i=1}^{n}\left(\lambda_{n i}^{(1)}+\frac{1}{B_{n}^{2}} \lambda_{n i}^{(2)}\right) \int_{T_{2} B_{n}}^{T B_{n}} e^{-t^{2} b_{n}^{2} /\left(2 B_{n}^{2}\right)} \frac{d t}{t} \\
& +\prod_{k=1}^{n}\left(\lambda_{n k}^{(1)}+\frac{1}{B_{n}^{2}} \lambda_{n k}^{(2)}\right) \int_{T_{2} B_{n}}^{T B_{n}} \frac{d t}{t} \\
\leq & C^{(3)}\left(\frac{\Lambda_{n}^{(1)}}{\bar{B}_{n}}+\Lambda_{n}^{(2)}\right) b_{n}^{-3}
\end{aligned}
$$

and (1) follows from (2) and (4)-(6).

\section{BIBLIOGRAPHY}

1. P. V. Slyusarchuk and I. Yo. Polyak, A generalization of a V. M. Zolotarev result, Naukovyi Visnyk Uzhgorod. Univer., Ser. Matem. 3 (1997), 184-189. (Ukrainian)

2. V. M. Zolotarev, A modern theory of summation of independent random variables, "Nauka", Moscow, 1986. (Russian) MR 89a:60003

Chair of Mathematical Analysis, Department of Mathematics, Uzhgorod National UniverSity, Pidgirna Street 46, Uzhgorod, Ukraine

E-mail address: poliak_ivan@mail.ru

Received 20/NOV/2002

Translated by OLEG KLESOV 